\title{
Dexamethasone release from photopolymerised PEGDA 700 for cochlea drug delivery
}

\begin{abstract}
Sustained local drug release and high local drug concentrations can be achieved by specially designed DDSs, which can be created for different applications. This results in therapeutic drug amounts at the site of action, simultaneously providing a very low drug amount in total.

Bodily compartments that contain liquids may be used to transport the drugs from DDS into the tissue, such as the cochlea with the perilymph in the case of cochlea implants. However, predominantly dry environments, such as the middle ear, are lacking such a medium but may deliver enough moisture for the use of swellable DDS through the surrounding mucus membranes. Therefore, DDS with new functionalities are needed to ensure a sustained drug release.
\end{abstract}

In this study, the release of dexamethasone out of a photopolymer system is presented. The system is built from UV-polymerized PEGDA followed by the incorporation of dexamethasone via swelling.

The drug release is tested in vitro with isotonic $\mathrm{NaCl}$ solution for specified time periods, showing two phases: a swelling phase and a release phase.

After the swelling phase the concentration of dexamethasone in the release medium was not controlled by diffusion, although sink conditions were ensured. In contrast, this system can be used to release the drug until equilibrium with a final medium concentration that is far below the solubility of dexamethasone.

Hence, such DDS may be useful for dexamethasone delivery into the cochlea through the round window membrane by ensuring a constant concentration in the perilymph.

\footnotetext{
*Corresponding author: Eickner Thomas: Institute for Biomedical Engineering (University Medical Center Rostock, University of Rostock), Friedrich-Barnewitz Straße 4, Rostock, Germany, thomas.eickner@uni-rostock.de

Michael Teske, Natalia Rekowska, Volkmar Senz, Klaus-Peter Schmitz, Niels Grabow, Institute for Biomedical Engineering (University Medical Center Rostock, University of Rostock), Rostock, Germany,
}

Keywords: drug release, dexamethasone, equilibrium controlled release, PEGDA, Photopolymer, swelling, cochlea drug delivery,

https://doi.org/10.1515/cdbme-2020-3021

\section{Introduction}

Drug Delivery Systems (DDS) are mostly designed to obtain a sustained drug release. Due to the local application of DDS a high local concentration is achieved resulting in therapeutic amounts of drug at the site of action, simultaneously providing a very low systemic drug load. Compared to other drug applications, such as tablets or intravenous application, the use of DDS should result in fewer side effects.

A few examples of environments containing liquids, that are responsible for transportation of the drugs away from DDS into the systemic route or to the tissue can be found. Among them well known are for instance the blood stream for drug eluting stents for the treatment of coronary heart disease and inside the cochlea with perilymph for cochlea implants [1, 2]. In predominantly dry environments, such as the middle ear the drug is delivered into the cochlea through the round window membrane or the paranasal sinuses. In such cases, novel types of DDS are needed, to ensure functionalities such as swelling with low amount of water for mobilizing incorporated drugs in order to obtain a sustained drug release.

\section{Materials and Methods}

\subsection{Preparation of PEGDA-Samples}

PEGDA $_{700}\left(\mathrm{M}_{\mathrm{w}} \sim 700 \mathrm{~g} / \mathrm{mol}\right)$ was mixed with Irgacure stock solution ( $\mathrm{c}=500 \mathrm{mg} / \mathrm{mL}$ in DMSO) in a ratio of $39: 1 \mathrm{v} / \mathrm{v}$. $30 \mu \mathrm{L}$ of the mixture were pipetted into moulds that were built inhouse and polymerized for $30 \mathrm{~min}$ in the UV chamber CL-1000L (UVP, USA) at $\lambda=365 \mathrm{~nm}$. Polymer discs with a diameter of $5 \mathrm{~mm}$ were obtained. Afterwards the discs were 
washed 6 times for $30 \mathrm{~min}$ with $0.5 \mathrm{~mL}$ with Acetonitrile (ACN) followed by 3 times for $30 \mathrm{~min}$ with $0.5 \mathrm{~mL}$ water. Afterwards they were dried overnight at $40^{\circ} \mathrm{C}$ in the vacuum [3]. Discs with comparable masses were chosen for subsequent processing steps.

\subsection{Incorporation of Dexamethasone}

For drug incorporation the dexamethasone was solved in ethanol to a final concentration of $\mathrm{c}=2 \mathrm{mg} / \mathrm{mL}$. Samples were allowed to swell in $1 \mathrm{~mL}$ of the drug solution for six hours. Then the solution was discarded and the residual solvent was evaporated at $40^{\circ} \mathrm{C}$ in the vacuum overnight.

\subsection{Dexamethasone Release}

For the drug release specified time periods in isotonic $\mathrm{NaCl}-$ solution were chosen. To cover the initial burst release, time periods from fifteen seconds up to two minutes were used on the first day. Three samples each were released in $2 \mathrm{~mL}$ and $4 \mathrm{~mL}$, respectively at room temperature. The release study was continued on a different day for practical reasons (table 1). Samples were stored without medium.

Table 1: time periods for drug release continued on different days.

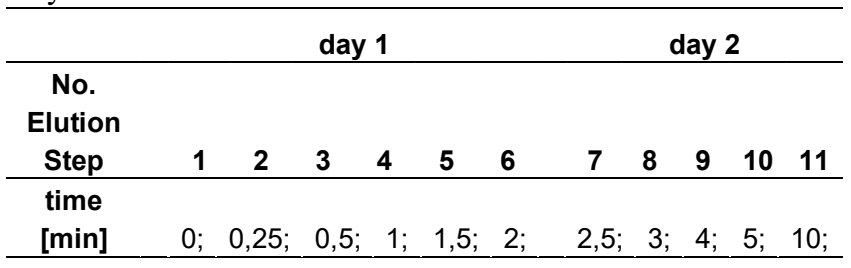

\section{Results and Discussion}

The release of dexamethasone was tested for specified time periods. During the first day only minor amounts of dexamethasone were released from the discs, resulting in a plateau at the beginning of the study as shown in figure 1 . Thus, no burst release was observed, as it would have been expected from such a hydrogel type DDS. Hence, a release mechanism different to a diffusion controlled one has to be supposed at least for the initial time period.

On the second day, the drug release increased dramatically (figure 1a: at $6.75 \mathrm{~min}$ ). The summarized time of release at the first day refers to as $4.25 \mathrm{~min}$. This is almost twice as long as the first time period at the second day with $2.5 \mathrm{~min}$. Thus, it can be assumed that a swelling controlled release mechanism [4] is existent. In fact this could be supported by the storage time between the two days of release testing. The swelling of the samples during storage was promoted by a small amount of $\mathrm{NaCl}$-solution that remained on the surface of the discs after the withdrawal of the medium.

Hence, the dexamethasone release exhibits two phases, a swelling phase and a release phase.

The use of $2 \mathrm{~mL}$ medium resulted in a faster drug release than the use of $4 \mathrm{~mL}$. As sink conditions were ensured, the reasons for that behaviour still remain unclear.
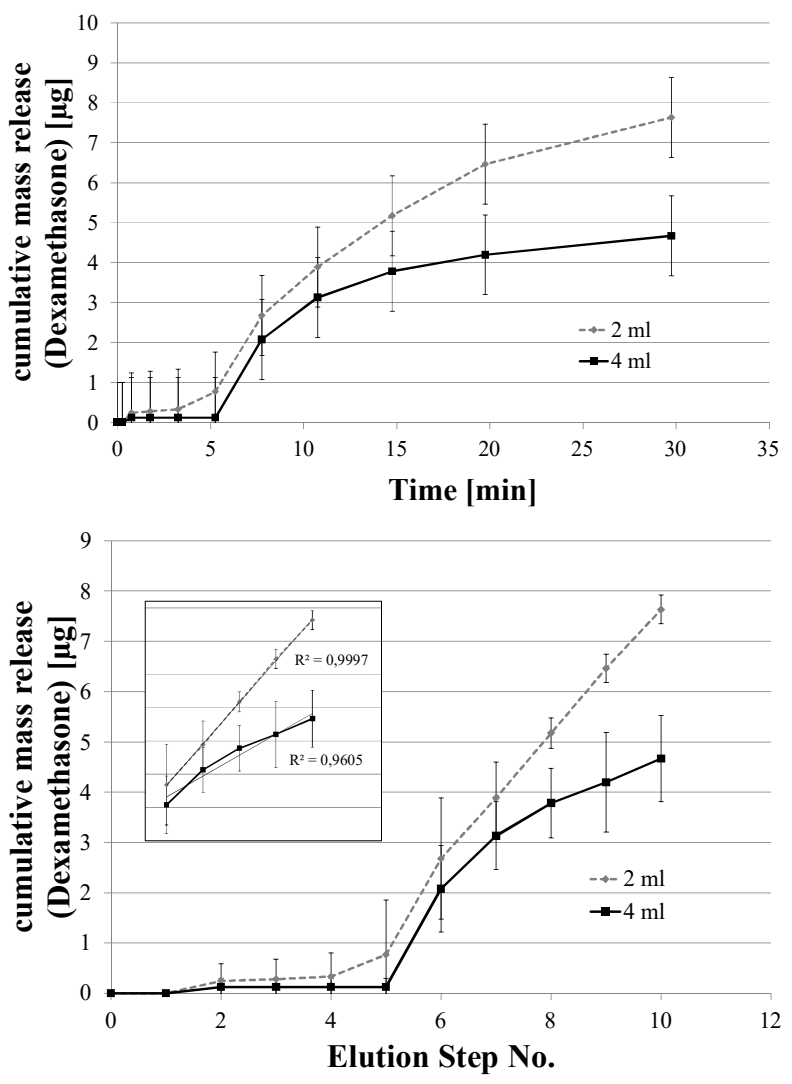

Figure 1: Cumulative release of dexamethasone, normalized to the used volume of medium ( $2 \mathrm{~mL}$ and $4 \mathrm{~mL}$ ), $\mathrm{n}=3$; a) according to time, b) according to the elution step. The swelling and the release phase are visible in a) and b). b) inlay: regression coefficients are shown, indicating the linear dependency on elution step for the release phase

\section{Conclusion}

In this study a drug release system, consisting of dexamethasone incorporated in photo polymerized PEGDA $_{700}$ is presented. The above presented drug release results show an equilibrium controlled release, which keeps a constant concentration in the surrounding medium. Such 
systems may be used in new pharmaceutical applications as drug delivery devices, such as the implants working through the round window membrane and ensuring a constant drug concentration in the perilymph. According to the specified properties a rapid initial burst release and a drug overdosing at the beginning of the therapy will be prevented.

Acknowledgment: The authors would like to thank Caroline Dudda for the excellent technical assistance.

\section{Author Statement}

Research funding: Financial support by the Federal Ministry of Education and Research (BMBF) within RESPONSE "Partnership for Innovation in Implant Technology" is gratefully acknowledged. Conflict of interest: Authors state no conflict of interest. Informed consent: Informed consent is not applicable. Ethical approval: The conducted research is not related to either human or animal use.

\section{References}

[1] Krenzlin S, Vincent C, Munzke L, Gnansia D, Siepmann F. Predictability of drug release from cochlear implants. Journal of Controlled Release. 2012;159:60-68.

[2] Acharya G, Park K. Mechanisms of controlled drug release from drug-eluting stents. Advanced Drug Delivery Reviews. 2006;58:287-401

[3] Rekowska N, Arbeiter D, Konasch D, Riess A, Mau R, Eickner T, Seitz H, Grabow N and Teske M,

Thermomechanical properties of PEGDA and its co-polymers Current Directions in Biomedical Engineering, 2018,4(1):669-672

[4] Langer R, New Methods of Drug delivery, Science 1990;249(4976):1527-1533. 